\title{
On elation Laguerre planes with a two-transitive orbit on the set of generators
}

\author{
Günter F. Steinke, \\ Markus J. Stroppel
}

\section{Stuttgarter}

Mathematische

Berichte

2017-009 
Fachbereich Mathematik

Fakultät Mathematik und Physik

Universität Stuttgart

Pfaffenwaldring 57

D-70 569 Stuttgart

E-Mail: preprints@mathematik.uni-stuttgart.de

WWW: http://www.mathematik.uni-stuttgart.de/preprints

\section{ISSN 1613-8309}

(C) Alle Rechte vorbehalten. Nachdruck nur mit Genehmigung des Autors.

LATEX-Style: Winfried Geis, Thomas Merkle, Jürgen Dippon 


\title{
On elation Laguerre planes with a two-transitive orbit on the set of generators
}

\author{
Günter F. Steinke, Markus J. Stroppel
}

\begin{abstract}
We study finite elation Laguerre planes with a group of automorphisms fixing a generator and acting two-transitively on the set of remaining generators. For odd order, this assumption characterizes the Miquelian Laguerre planes, but there are non-Miquelian examples if the order is even.
\end{abstract}

MSC 2010: 51B15, 51E25, 20B20.

Keywords: Laguerre plane, elation group, two-transitive group, socle, ovoidal Laguerre plane, Miquelian Laguerre plane.

\section{Introduction}

Laguerre planes generalize the geometry of non-trivial plane intersections of a cone over some oval in a projective plane; these intersections are referred to as circles. If the circles of a Laguerre plane are indeed obtained in this way then the Laguerre plane is called ovoidal. Miquel's theorem holds in the (essentially unique) ovoidal Laguerre plane over an oval quadric in the plane over a field $F$. Conversely, the class of such Laguerre planes is characterized by Miquel's theorem, and these planes are called Miquelian (or classical).

All finite Laguerre planes known to date are ovoidal; they are even Miquelian if their order is odd. However, it is not clear whether this state of knowledge is due only to the fact that appropriate constructions have not yet been found. Elation Laguerre planes (as introduced in [37] and [29], see Section 1 below) appear to play a role similar to translation planes among the projective planes. Several constructions are known of (infinite) non-Miquelian elation Laguerre planes.

It has been shown in [34] and [35] that every finite elation Laguerre plane with a group of automorphisms acting two-transitively on the set of generators is Miquelian. A similar result (under weaker geometric assumptions, but for non-solvable groups only) has been obtained in [2]. In the present paper, we consider a finite elation Laguerre plane $\mathscr{L}$ of order $q$, and assume that there exists a group of automorphisms fixing one generator $[\infty]$ and acting two-transitively on the set of generators different from $[\infty]$. This is equivalent to the existence of a subgroup $\Gamma \leq \operatorname{Aut}(\mathscr{L})$ that fixes some incident point-circle pair $(\infty, K)$ and acts two-transitively on $H:=K-\{\infty\}$. Such a situation can be found in each Miquelian Laguerre plane; there are also some non-Miquelian examples (see Section 6 below).

\footnotetext{
* This research was supported by a Visiting Erskine Fellowship from the University of Canterbury for the second author.
} 
For odd $q$, we then show that $\mathscr{L}$ is the Miquelian Laguerre plane over $\mathbb{F}_{q}$. If $q$ is even then $\mathscr{L}$ is a translation Laguerre plane (in the sense of [12]), and the derived affine plane $\mathbb{A}_{\infty}$ at $\infty$ is a plane over a generalized twisted field if $q \neq 2^{6}$. If $\mathrm{A}_{\infty}$ is Desarguesian then $\mathscr{L}$ is ovoidal over a translation oval, and known explicitly. In particular, this is the case when $q=2^{e}$ and $e$ is a prime or a square of a prime.

In Section 1 we collect basic facts we need on finite elation Laguerre planes and their automorphisms. The next section deals with the socle of the two-transitive group $G$ induced by $\Gamma$ on $H$ and excludes non-abelian socles, so $G$ is of affine type. As the crucial step for the main result we show in Section 3 that the projective closure of $\mathbb{A}_{\infty}$ has Lenz type at least $V$ and thus can be coordinatized over a semifield. The following Section 4 examines the case of odd order; we find sufficiently many shift groups to secure that the affine plane $\mathbb{A}_{\infty}$ is Desarguesian (whence $\mathscr{L}$ is Miquelian). In Section 5 we show that the stabilizer $G_{o}$ of a point $o \in H$ is isomorphic to a subgroup of $\operatorname{P\Gamma L}(1, q)$. The last section discusses the case of even order and completely determines the Laguerre planes when $\mathbb{A}_{\infty}$ is Desarguesian.

\section{Elation Laguerre planes}

We briefly indicate basic information about elation Laguerre planes in the sequel, and refer the reader to the more detailed introduction in [34].

Axioms for Laguerre planes. A finite Laguerre plane $\mathscr{L}=(P, \mathscr{C}, \mathscr{G})$ of order $n$ consists of a set $P$ of $n(n+1)$ points, a set $\mathscr{C}$ of $n^{3}$ circles and a set $\mathscr{G}$ of $n+1$ generators, where both circles and generators are subsets of $P$, such that the following three axioms are satisfied.

(G) $\mathscr{G}$ partitions $P$, each generator contains $n$ points, and there are $n+1$ generators.

(C) Each circle intersects each generator in precisely one point.

(J) Three points no two of which are on the same generator are joined by a unique circle.

Circles through $x$ are called touching in $x$ if they are equal or have no other point in common. The derived affine plane $\mathbb{A}_{x}$ at a point $x \in P$ has the collection of all points not on the generator $[x]$ through $x$ as point set and, as lines, all circles passing through $x$ (without the point $x$ ) and all generators apart from $[x]$. The axioms above easily yield that $\mathbb{A}_{x}$ is an affine plane, indeed. We refer to the generators as vertical lines in $\mathbb{A}_{x}$. The set of non-vertical lines is induced from the set $\mathscr{C}_{x}$ of all circles passing through $x$. Circles that touch each other in $x$ give parallel lines in $\mathbb{A}_{x}$. The projective completion of $\mathbb{A}_{x}$ will be denoted by $\mathbb{P}_{x}$.

The group $\operatorname{Aut}(\mathscr{L})$ of a Laguerre plane $\mathscr{L}$ acts on the set $\mathscr{G}$ of generators. We call $\mathscr{L}$ an elation Laguerre plane if the kernel $\Delta$ of that action acts transitively on the set $\mathscr{C}$ of circles. It is known (see [29, 1.3]) that in every finite elation Laguerre plane the group $\Delta$ has a (unique) regular normal subgroup $E$; this group will be called the elation group. It is also known that $E$ is elementary abelian and acts regularly on $\mathscr{C}$.

Throughout the present paper, we study an elation Laguerre plane $\mathscr{L}=(P, \mathscr{C}, \mathscr{G})$ admitting a group $\Gamma \leq \operatorname{Aut}(\mathscr{L})$ that fixes some incident point-circle pair $(\infty, K)$ and acts two-transitively on $K-\{\infty\}$. We abbreviate $H:=K-\{\infty\}$; this set will be considered as a ("horizontal") line in the derived affine plane $\mathbb{A}_{\infty}$. 
1.1 Theorem ([7], [26], [29], [30], [31], [34]). Let $\mathscr{L}$ be an elation Laguerre plane of finite order $q$, with elation group $E$. We consider a point $x$ and the derived projective plane $\mathbb{P}_{x}$.

1. The projective plane $\mathbb{P}_{x}$ is a dual translation plane; the translation center is the point $\omega$ at infinity of vertical lines (induced by generators of the Laguerre plane), and $E_{x}$ induces the full dual translation group. Furthermore $E_{x}$ acts trivially on $[x]$.

2. The order $q$ is a prime power, say $q=r^{e}$ with a prime $r$ and some integer $e$.

3. If $q$ is a prime then $\mathscr{L}$ is Miquelian.

If $q$ is the square of a prime and $q$ divides the order of the elation complement (i.e., if $q$ divides the order of the stabilizer of some circle through $x$ ) then $\mathscr{L}$ is Miquelian.

Any Laguerre plane of order at most ten is ovoidal and, in fact, Miquelian except in case of order eight.

4. Each circle not through $x$ induces an oval in $\mathbb{P}_{x}$. That oval passes through the translation center $\omega$ and has the line at infinity as a tangent.

1.2 Lemma. In the automorphism group of the Miquelian Laguerre plane of order $q=r^{e}$, the stabilizer of any circle $C$ is isomorphic to the direct product $\mathrm{P} \Gamma \mathrm{L}(2, q) \times \mathrm{C}_{q-1}$ and induces a group isomorphic to $\mathrm{P} \Gamma \mathrm{L}(2, q)$ on $C$. The action on $\mathscr{C}$ is equivalent to the natural three-transitive action on the projective line over $\mathbb{F}_{q}$, while the kernel of the restriction map is cyclic of order $q-1$. In particular, the stabilizer of any incident point-circle pair $(x, C)$ is solvable, and two-transitive on $C-\{x\}$. Every regular abelian normal subgroup of that stabilizer acts trivially on $[x]$.

In any ovoidal Laguerre plane of order $q$, the stabilizer of any incident point-circle pair $(x, C)$ is solvable, in fact isomorphic to a subgroup of a triangular subgroup of $\mathrm{P \Gamma L}(3, q)$.

1.3 Lemma. Let $\mathscr{L}$ be a Laguerre plane of finite order $q$, and let $\sigma$ be an automorphism of $\mathscr{L}$ fixing some circle C.

1. If $\sigma$ is an involution, then one of the following holds.

(a) $\sigma$ fixes every point on $C$; then $q$ is odd, and $\sigma$ induces an axial involution on $\mathbb{A}_{x}$, for each $x \in C$.

(b) The order $q$ is a square and $\sigma$ fixes precisely $1+\sqrt{q}$ points on $C$; then $\sigma$ induces a Baer involution on $\mathbb{A}_{x}$, for each fixed point $x$ on $C$.

(c) $\sigma$ fixes at most two points on $C$. If there is a fixed point $x$ on $C$ then $\sigma$ induces an axial involution on $\mathbb{P}_{x}$.

2. If $\mathscr{L}$ is an elation Laguerre plane and $\sigma$ acts as an involution on $C$ fixing more than two points on $C$ (but not all of them), then $q$ is a square.

Proof. The claims in assertion 1 are proved in [35, 2.2].

Now assume that $\sigma$ induces an involution on $C$, and that $\sigma$ fixes more than two points on $C$. Let $\infty$ be one of the fixed points. Then $\sigma$ induces a collineation $\sigma^{\prime}$ of the dual of the derived projective plane $\mathbb{P}_{\infty}$, and $\sigma^{\prime}$ fixes at least three points on the translation axis (corresponding to generators through the fixed points on $C$ ) of that translation plane. As $\sigma^{\prime}$ induces an involution on the translation axis, the order $q$ must be a square by [9, Theorem 2]. 
Involutions fixing a circle but no point on it occur, for instance, in Miquelian planes of order $q \equiv 3(\bmod 4)$ (because then $\operatorname{PSL}(2, q)$ contains involutions fixing no point of the projective line). Involutions of the kind discussed in 1.3 (a) are well understood in elation Laguerre planes:

1.4 Lemma ([29, Thm. $2 \mathrm{~d})])$. If $\mathscr{L}$ is a finite elation Laguerre plane of odd order then at each circle $C$ there exists a unique reflection, i.e., an involutory automorphism of $\mathscr{L}$ fixing each point on $C$ (and no others).

In particular, if $\Delta_{C}$ contains an involution then that involution is uniquely determined by $C$, and centralized by the stabilizer of $C$ in $\operatorname{Aut}(\mathscr{L})$.

1.5 Lemma. Let $\mathscr{L}$ be a finite Laguerre plane of order $q$ and let $S \leq \operatorname{Aut}(\mathscr{L})$ be of odd order. If $S$ fixes more than q circles then $S \leq \Delta$; that is, the group $S$ fixes each generator of $\mathscr{L}$.

Proof. Let $[x]$ be any generator of $\mathscr{L}$. Since $[x]$ has $q$ points, there must be at least two distinct circles fixed by $S$ that intersect $[x]$ in the same point $w$. The intersection of these two circles is a set $\left\{w, w^{\prime}\right\}$ of size at most 2 , and invariant under $S$. But the orbit $S(w)$ of $w$ under $S$ has odd length. Thus $S$ fixes $w$ and then also the generator $[w]=[x]$.

1.6 Lemma. Let $\mathscr{L}=(P, \mathscr{C}, \mathscr{G})$ be a finite Laguerre plane and let $S \leq \operatorname{Aut}(\mathscr{L})$. If $S$ fixes at least three points on a circle and a fourth point off this circle, then the geometry $\mathscr{L}_{S}=\left(P_{S}, \mathscr{C}_{S}, \mathscr{G}_{S}\right)$ of all fixed points $P_{S}$, all fixed circles $\mathscr{C}_{S}$ and all fixed generators $\mathscr{G}_{S}$ of $S$ is a Laguerre plane. In particular, any two fixed circles of $S$ intersect in fixed points (if they meet at all).

Proof. Axiom (C): Each circle in $\mathscr{C}_{S}$ intersects each generator in $\mathscr{G}_{S}$ in precisely one point which also belongs to $P_{S}$.

Axiom (J): Any three points in $P_{S}$ no two of which are on the same generator are joined by a unique circle which then belongs to $\mathscr{C}_{S}$.

Axiom (G): Let $n+1$ be the number of generators in $\mathscr{G}_{S}$. Our assumptions secure $n \geq 3$. For any three generators in $\mathscr{G}_{S}$, take their intersections $x, y$, and $z$, respectively, with some fixed circle $C \in$ $\mathscr{C}_{S}$. Each circle touching $C$ in $z$ meets both $[x]$ and $[y]$, and we obtain a bijection from $[x] \cap P_{S}$ onto $[y] \cap P_{S}$. Therefore, the number $m$ of fixed points on a fixed generator is constant, and $m \geq 3$.

Choose a fourth point $w \in([x]-\{x\}) \cap P_{S}$. If $v \in P_{S} \cap C$ and $v \neq x, y$, then the circle through $w$, $y$ and $v$ is fixed by $S$ and so is its intersection with $[z]$. Furthermore, the circle through $w$ that touches $C$ at $y$ is fixed by $S$ and so is its intersection with $[z]$. All these points of intersection are mutually distinct so that $m \geq n$.

We can form $m$ circles through $w, y$ and one of the fixed points on $[z]$. One of these touches $C$ in $y$, but each of the other $m-1$ circles has a second point $u_{w} \neq x, y$ in common with $C$. But then $u_{w}$ is also fixed. In this way we obtain $m+1 \geq n+1$ fixed points on $C$, and $m=n$ follows. Thus Axiom (G) is established, and $\mathscr{L}_{S}$ is a Laguerre plane (of order $m$ ), as claimed.

There do exist involutions on Laguerre planes fixing two circles but moving their intersection points:

1.7 Example. Let $\alpha$ denote the generator of the Galois group Gal $\left(\mathbb{F}_{s^{2}} / \mathbb{F}_{s}\right)$. Then $\gamma$ given in affine coordinates by $\gamma(x, y)=(\alpha(x), \alpha(y))$ is an involutory automorphism of the Miquelian Laguerre plane $\mathscr{M}\left(s^{2}\right)$ of order $s^{2}$.

The traces of circles of $\mathscr{M}\left(s^{2}\right)$ are just the graphs of polynomials of degree at most two over $\mathbb{F}_{s^{2}}$. Such a circle is fixed by $\gamma$ precisely if the polynomial has coefficients in $\mathbb{F}_{s}$. 
Let $f(x) \in \mathbb{F}_{s}[x]$ be a quadratic irreducible polynomial over $\mathbb{F}_{s}$, and let $C$ be the circle of $\mathscr{M}\left(s^{2}\right)$ whose trace is the graph of $f$. So both $C$ and the circle $X$ with trace $\left\{(x, 0) \mid x \in \mathbb{F}_{s^{2}}\right\}$ are fixed by $\gamma$. However, the two points in $C \cap X$ are interchanged by $\gamma$; in fact, the intersection consists of the two solutions of $f(x)=0$ in $\mathbb{F}_{s^{2}} \backslash \mathbb{F}_{s}$.

The following straightforward result (see [24, Lemma 19.3]) will be helpful.

1.8 Lemma. Let $r$ and $s$ be primes and $e, f$ be positive integers such that $r^{e}+1=s^{f}$. Then one of the following holds:

1. $s=2, e=1$ ( $r$ is a Mersenne prime);

2. $r=2, f=1$ (s is a Fermat prime);

3. $r=2, e=3, s=3, f=2$.

1.9 Lemma. Let $\mathscr{L}$ be any Laguerre plane, and consider the set $\operatorname{Fix}_{\mathscr{C}}(\alpha)$ of fixed circles for $\alpha \epsilon$ Aut $(\mathscr{L})$. Assume that $\alpha$ has order 3 and fixes precisely two generators $[a]$ and $[b]$. If Fix $_{\mathscr{C}}(\alpha)$ contains two circles in the bundle $\mathscr{C}_{a} \cap \mathscr{C}_{b}$ then $\operatorname{Fix}_{\mathscr{C}}(\alpha)$ is that bundle.

Proof. If $g$ is a generator different from $[a]$ and from $[b]$ then there is a bijection from $g$ onto Fix $_{\mathscr{C}}(\alpha)$ mapping $x \in g$ to the circle $C_{x}$ through the points $x, \alpha(x)$ and $\alpha^{2}(x)$. Since $\alpha$ has order 3, it follows that $C_{x} \cap C_{y}$ consists of fixed points of $\alpha$ for all $x \neq y$. Hence $C_{x} \cap C_{y} \subset[a] \cup[b]$.

Let $x, y, z \in g$ be three distinct points and assume that $C_{x} \cap C_{y}=\{a, b\}$. Suppose that $C_{x} \cap C_{z}=\{a\}$. Then $C_{x}, C_{y}, C_{z}$ induce lines of the derived affine plane $\mathbb{A}_{a}$. But $C_{x}$ and $C_{z}$ are parallel in $\mathbb{A}_{a}$ whereas $C_{x}$ and $C_{y}$ are not. Hence $C_{y}$ and $C_{z}$ intersect in an affine point $s$ not on [o], leading to the contradiction that $s$ and thus $[s]$ are fixed by $\alpha$.

Now suppose that both $C_{x} \cap C_{z}$ and $C_{y} \cap C_{z}$ are empty. We consider the derived projective plane $\mathbb{P}_{a}$. In this plane $\alpha$ induces a collineation, $C_{x}$ and $C_{y}$ induce nonparallel lines and $C_{z}$ induces an oval that has $C_{x}$ and $C_{y}$ as exterior lines. The oval has a unique tangent at $C_{z} \cap[b]$. This tangent is fixed by $\alpha$ and so are its points of intersection with $C_{x}$ and $C_{y}$. At least one of these points of intersection is an affine point not on $[b]$ and we have again a contradiction.

This shows that $C_{x} \cap C_{z}=C_{y} \cap C_{z}=C_{x} \cap C_{y}=\{a, b\}$. Hence $\operatorname{Fix}_{\mathscr{C}}(\alpha)$ consists of the bundle of circles through $a$ and $b$.

1.10 Lemma. Let $\mathscr{L}$ be a finite Laguerre plane of even order. Let $\alpha \in \operatorname{Aut}(\mathscr{L})$ be an automorphism of order 3 that fixes precisely two generators $[\infty]$ and $[o]$. Then $\operatorname{Fix}_{\mathscr{C}}(\alpha)$ is precisely one of the following:

- the bundle of circles through two points $a \in[\infty]$ and $b \in[o]$;

- the bundle of circles touching at a point of $[\infty]$ or of $[o]$.

Proof. We keep the notation from the proof of Lemma 1.9. Let $x, y, z \in g$ be three distinct points. By Lemma 1.9 we may assume the circles $C_{x}, C_{y}, C_{z}$ pairwise intersect in at most one point.

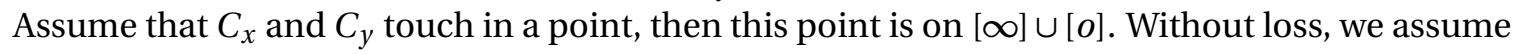
that $C_{x} \cap C_{y}=\{a\}$ where $a \in[\infty]$. Suppose that $C_{x} \cap C_{z}=\{b\}$ where $b \in[o]$. Since $C_{z}$ does not pass through $a$, it induces an oval in $\mathbb{P}_{a}$. Furthermore, $C_{x}$ induces a tangent to this oval. Since $\mathscr{L}$ has even order, the oval has a knot $k$ which lies on the line at infinity because that line is a tangent. Since $C_{x}$ and $C_{y}$ induce parallel lines, $C_{y}$ induces another tangent. Thus $C_{y}$ and $C_{z}$ intersect in an affine point not on $[o]$ - a contradiction. 
Now suppose that both $C_{x} \cap C_{z}=$ and $C_{y} \cap C_{z}$ are empty. As before $C_{z}$ induces in $\mathbb{P}_{a}$ an oval that now has $C_{x}$ and $C_{y}$ as exterior lines. Moreover, $C_{x}$ and $C_{y}$ do not pass through the knot of the oval. The unique tangent to the oval at $C_{z} \cap[o]$ therefore meets $C_{x}$ and $C_{y}$ in affine points - a contradiction.

This shows that $C_{x} \cap C_{z}=C_{y} \cap C_{z}=C_{x} \cap C_{y}=\{a\}$. Hence Fix $\mathscr{C}(\alpha)$ consists of the bundle of circles touching at $a$ if it contains two circles in this bundle.

Finally assume that $C_{x} \cap C_{y}$ is empty. The previous case and Lemma 1.9 show that then also $C_{x} \cap C_{z}$ and $C_{y} \cap C_{z}$ are empty. Hence $\operatorname{Fix}_{\mathscr{C}}(\alpha)$ consists of a flock if it contains two disjoint circles. This implies that $\alpha$ fixes the generators [ $\infty]$ and [o] pointwise. By [23, Lemma 3.1] an automorphism of a Laguerre plane that induces the identity on two different generators is an involution or belongs to the kernel. Hence, this configuration cannot occur by the assumptions made on $\alpha$.

\section{The regular normal subgroup}

The socle of a finite group is defined as the (characteristic) subgroup generated by all minimal normal subgroups. By a theorem due to Burnside (see [4, Ch.X, Thm. XIII, p. 202]) the socle $M$ of the two-transitive group $G$ induced by $\Gamma$ on $H$ is transitive on $H$, and either simple or elementary abelian.

If $M$ is simple, we let $\tilde{M}$ denote the stationary term in the commutator series of the pre-image of $M$ under the quotient map from $\Gamma$ onto $G$. Then $\tilde{M}$ is a perfect central extension of $M$ and the center of $\tilde{M}$ is a quotient of the Schur multiplier of $M$ (cf. [35, 3.3]).

The classification of finite simple groups yields a list of the possibilities for a simple socle, see Table 1. This list is adapted from [5] and [21]; note that $\mathrm{A}_{q}$ for $q=5$ also occurs in the guise of $\operatorname{PSL}(2, f)$ on $\left(f^{2}-1\right) /(f-1)$ for $f=4$. We have modified the names for the parameters to avoid confusion with our fixed meaning for $q$. Also, we use the order $f^{2}$ of the quadratic extension field for the unitary groups.

\begin{tabular}{|l|c|l|}
\hline$M$ & $q$ & remarks/restrictions \\
\hline $\mathrm{A}_{q}$ & $q$ & $q \geq 5, \quad$ (two representations if $q=6)$ \\
$\operatorname{PSL}(d, f)$ & $\left(f^{d}-1\right) /(f-1)$ & $\begin{array}{l}d \geq 2, \quad(d, f) \notin\{(2,2),(2,3)\} \\
\text { (two representations if } d>2)\end{array}$ \\
$\operatorname{PSU}\left(3, f^{2}\right)$ & $f^{3}+1$ & $f>2$ \\
$\mathrm{Sz}\left(2^{2 a+1}\right)$ & $2^{4 a+2}+1$ & $a>0$, Suzuki groups: ${ }^{2} \mathrm{~B}_{2}\left(2^{2 a+1}\right)$ \\
$\mathrm{R}\left(3^{2 a+1}\right)$ & $3^{6 a+3}+1$ & $a>0$, Ree groups: ${ }^{2} \mathrm{G}_{2}\left(3^{2 a+1}\right)$ \\
$\operatorname{PSp}(2 d, 2)$ & $2^{2 d-1} \pm 2^{d-1}$ & $d>2$ \\
$\operatorname{PSL}(2,11)$ & 11 & (two representations) \\
$\mathrm{A}_{7}$ & 15 & (two representations) \\
$\operatorname{PSL}(2,8)$ & 28 & socle of $\mathrm{R}(3)$ \\
$\mathrm{M}_{n}$ & $n$ & Mathieu groups, $n \in\{11,12,22,23,24\}$ \\
& & (two representations for $n=12)$ \\
$\mathrm{M}_{11}$ & 12 & Mathieu group \\
$\mathrm{Co}{ }_{3}$ & 276 & Conway group \\
$\mathrm{HS}$ & 176 & Higman-Sims group (two representations) \\
\hline
\end{tabular}

Table 1: Non-abelian socles of two-transitive groups 


\subsection{Lemma. If $q>4$ then the socle $M$ is not isomorphic to $\mathrm{A}_{q}$.}

Proof. Elation Laguerre planes of order at most 9 are ovoidal (1.1]3 see [7]), and the stabilizer of any point-circle flag is solvable (see 1.2. As $\mathrm{A}_{q}$ is not solvable for $q>4$, we may assume $q>9$ here. Therefore, the Schur multiplier of $\mathrm{A}_{q}$ is cyclic of order 2 (cf. [1, (33.15)]). If $\tilde{M}$ contains a central involution acting trivially on $H$ then $q$ is odd and this central involution is the unique reflection $\zeta$ at $K$, see 1.4. In order to avoid a case distinction, we consider the group $\Psi=\langle\zeta\rangle \tilde{M}$, where $\zeta:=$ id if $q$ is even.

The stabilizer $\Psi_{o}$ of a point $o \in H$ contains $\zeta$ and induces a group isomorphic to $\mathrm{A}_{q-1}$ on $H$. Therefore, it is a group of order $(q-1) !=\left|S_{q-1}\right|$ but not isomorphic to $S_{q-1}$. The action of $\Psi_{o}$ on the $q-1$ points of $[o]-\{o\}$ is thus not faithful. The kernel $\Xi$ of this action does not contain $\zeta$, and restriction to $H$ gives an injective homomorphism onto a normal subgroup of $\mathrm{A}_{q-1}$. The latter is simple, and $\Xi \cong \mathrm{A}_{q-1}$ follows.

The subgroup $\Xi \cong \mathrm{A}_{q-1}$ of $\Psi$ acts in the usual way on the $q-1$ points of $H-\{o\}$, and therefore contains involutions that fix $q-4$ points of $H$. These involutions must be Baer involutions of the affine plane $\mathbb{A}_{\infty}$, and we obtain the equation $(q-4)^{2}=q$. As this equation has no integer solutions, we have reached a contradiction.

\subsection{Lemma. If $q>3$ then the socle $M$ is not isomorphic to $\operatorname{PSL}(d, f)$.}

Proof. Aiming at a contradiction, we assume $M \cong \operatorname{PSL}(d, f)$. Then $r^{e}=q=\left(f^{d}-1\right) /(f-1)$.

If $d=2$ then we know from 1.8 that either $r=2$ and $f=2^{m}-1$ is a Mersenne prime (with odd $m$ ), or $e=1$ and $q=r=f+1$ is a Fermat prime, or $q=9$.

For $q=9$ or $q$ prime we know that $\mathscr{L}$ is Miquelian, and $M$ is embedded in a two-point stabilizer in $\operatorname{P\Gamma L}(2, q)$. Then solvability of that stabilizer (see 1.2 implies $q \leq 3$.

Assume that $f=2^{m}-1$ is a Mersenne prime. Then $m$ is odd, and $f \equiv 1(\bmod 4)$. Therefore, each involution in $\operatorname{PSL}(2, f)$ fixes two points in $H$ and then three points in $K$. Applying 1.32 we find that the order $q=f+1=2^{m}$ must be a square, contradicting the fact that $m$ is odd.

It remains to treat the cases where $d \geq 3$. If $f$ is odd then the Schur multiplier of $\operatorname{PSL}(d, f)$ has order 2 (see [18, 25.7], cf. [1, 3.3.6]), and $\tilde{M}$ is covered by $\operatorname{SL}(d, f)$. We consider an involution $J \in$ $\operatorname{SL}(d, f)$ such that the fixed points in $\left(\mathbb{F}_{f}\right)^{d}$ form a subspace of codimension 2. The eigenspaces of $J$ then have dimensions $d-2$ and 2, respectively, and $J$ induces an involution on $\mathbb{A}_{\infty}$ fixing $\left(f^{d-2}-1+f^{2}-1\right) /(f-1)$ points of the affine line $H$. As $J$ must induce a Baer involution on the projective completion of $\mathbb{A}_{\infty}$, we obtain $\left(f^{d-2}+f^{2}-2\right)^{2}=\left(f^{d}-1\right)(f-1)$. Now $(-2)^{2} \equiv(-1)^{2}(\bmod f)$ yields $f=3$. Writing $X:=3^{d-2}$ we obtain the quadratic equation $X^{2}-4 X+51=0$. This equation has no real solution, let alone an integer one.

If $f=2^{m}$ is even then the Schur multiplier is trivial unless $(d, f) \in\{(3,4),(4,2)\}$ (see [18, 25.7], cf. [1, 3.3.6]). The group $\operatorname{SL}(d, f) \cong \operatorname{PSL}(d, f)$ contains an involution $J$ fixing a hyperplane pointwise. Again, this involution must induce a Baer involution on the projective closure of $\mathbb{A}_{\infty}$. Then $\left(f^{d-1}-1\right)^{2}=\left(f^{d}-1\right)(f-1)$ follows, leading to the contradiction $f \equiv 0(\bmod 2 f)$. The groups $\operatorname{PSL}(d, f)$ with $(d, f) \in\{(3,4),(4,2)\}$ have their two-transitive actions on 21 and 15 points, respectively. These are not prime powers, and cannot be orders of elation Laguerre planes.

\subsection{Theorem. The socle of $G$ is abelian.}

Proof. We have to discuss the entries of Table 1. The groups $\mathrm{A}_{q}$ (for $q>4$ ) and $\operatorname{PSL}(d, f)$ in their two-transitive representation (for $d>1$ ) have been excluded by 2.1 and 2.2 respectively.

Clearly, all two-transitive groups with degrees divisible by more than one prime are excluded by the observation that $q$ is a prime power (cf.1.1). 
Every elation Laguerre plane of prime order is Miquelian. No simple group fixes an incident point-circle pair in a Miquelian plane, see 1.2 . This observation excludes all non-abelian socles of two-transitive groups of prime degree from our list.

We exclude the remaining non-abelian candidates for socles (cf. Table1) one by one.

$\operatorname{PSU}\left(3, f^{2}\right)$ : We have $r^{e}=q=f^{3}+1$ here. The only solutions for this equation are $q=9$ or $q$ a Fermat prime (as $f$ is a prime power, cf. 1.8). In these cases, the Laguerre plane is Miquelian (see 1.13), and 1.2 leads to a contradiction.

$\mathrm{Sz}\left(2^{2 a+1}\right)$ : Here $r^{e}=q=2^{4 a+2}+1$, we have $e=1$ by 1.8 the Laguerre plane $\mathscr{L}$ is Miquelian, and 1.2 leads to a contradiction.

$\mathrm{R}\left(3^{2 a+1}\right)$ : Then $r^{e}=q=3^{6 a+3}+1$, which is impossible by 1.8 .

2.4 Remark. One can define a Suzuki group $\mathrm{Sz}(2)$, i.e., $\mathrm{Sz}\left(2^{a}\right)$ for $a=0$. This gives a two-transitive group of order 20 and degree 5 . However, that group is not simple, but isomorphic to AGL $(1,5)$. The first part of the argument in the proof of 2.3 remains valid but we do not arrive at a contradiction because $\mathrm{Sz}(2) \cong \mathrm{AGL}(1,5)$ is isomorphic to the stabilizer of an incident point-circle pair in the automorphism group of the Miquelian plane of order 5, cf. 1.2

\section{Semifield planes}

Before we prove the main result of this section, we establish a result needed in the proof of 3.2 below. There are more cases of two-transitive groups of affine type to be studied in Section 5 below, but we need this particular case for the proof of 3.2 . Afterwards, we will use 3.2 (via its corollary 3.3) in order to exclude several cases in Section 5

3.1 Theorem. Assume that $G_{o}$ has a normal subgroup isomorphic to $\operatorname{SL}(d, f)$ or to $\operatorname{Sp}(d, f)$, where $q=f^{d}$ for some $d>1$. Then $d=2=f$; the plane is then the Miquelian plane of order 4 , and $G \cong \mathrm{S}_{4} \cong \mathrm{A} \Gamma \mathrm{L}(1,4) \cong \mathrm{AGL}(2,2)$.

Proof. Let $S \unlhd G_{o}$ be such a normal subgroup, and let $\tau$ be a transvection (cf. [18, 6.4, 9.17]) in $S$. Let $f=r^{c}$ for the prime $r$, and pick a Sylow $r$-subgroup $R$ of the pre-image in $\Gamma_{o}$ of $\langle\tau\rangle$. Then $R$ acts faithfully on $H$ because the order of $\Delta_{K}$ divides $q-1$. Therefore, some element $\rho \in \Gamma_{o}$ of order $r$ induces $\tau$ on $H$. Note that $\rho$ fixes $f^{d-1}$ points in $H$.

Assume first that $r$ is odd. The incidence structure $\mathscr{F}$ consisting of the fixed points, fixed generators and fixed circles of $\rho$ is a Laguerre (sub-)plane (see 1.6); the order of $\mathscr{F}$ is $f^{(d-1)}$. Therefore, we have $f^{3(d-1)}$ circles in $\mathscr{F}$. As $f^{3(d-1)}>f^{d}$ we obtain that each generator contains a point on at least two fixed circles. Thus $\rho$ fixes each generator, and then each point on $K$. This contradiction excludes the case $r>2$.

If $r=2$ then $f^{d-1}>1$ implies that $\rho$ is a Baer involution. This yields $f^{d}=q=f^{2(d-1)}$, and $d=2$ follows. Thus $S \cong \mathrm{SL}(2, f)=\mathrm{Sp}(2, f)$. We further assume $f>2$ and consider a Sylow 2-subgroup $T$ of the pre-image $\pi^{-1}(S)$. Since $\Delta_{K}$ has odd order, the image $\pi(T) \cong T$ is a Sylow 2-subgroup of $S$, and has order $f \geq 4$. Now $T$ fixes precisely $f+1$ generators of $\mathscr{L}$, and fixes at least 2 points on each such generator. The collection of fixed points, fixed circles and fixed generators forms a Laguerre plane $\mathscr{L}_{T}$ of order $f$, cf. 1.6 . Thus $T$ fixes $f^{3}$ circles.

Consider $x \in K-P_{T}$. Since $f^{3}>f^{2}=q$, there must be at least two distinct circles of $\mathscr{L}_{T}$ that intersect $[x]$ in the same point $w$. The intersection of these two circles is a set $\left\{w, w^{\prime}\right\}$ of size at most 2 , 
and invariant under $T$. Hence the stabilizer $T_{w}$ has order at least $\frac{f}{2} \geq 2$. Thus $T_{w}$ is nontrivial, fixes the generator $[x]$ and then also $x$. In the derived affine plane at $x$ each involution in $T_{w}$ fixes more than $f$ points on $K$ and also points off $K$, which is impossible.

3.2 Theorem. The projective closure $\mathbb{P}_{\infty}$ of the affine plane $\mathbb{A}_{\infty}$ has Lenz type at least $V$. The group of translations along $H$ is induced by a normal subgroup $\tilde{M}$ of order $q$ in $\Gamma$, and $\tilde{M}$ induces the regular normal subgroup $M$ in $G$.

Proof. The assertions are clear if $\mathscr{L}$ is Miquelian, cf.1.2. So assume that $\mathscr{L}$ is not Miquelian.

We know from 2.3 that the socle $M$ of the group $G$ induced by $\Gamma$ on $H$ is a regular abelian normal subgroup. So $M$ is elementary abelian, and has order $q=r^{e}$. Let $\tilde{R}$ be a Sylow $r$-subgroup of $\Gamma$. As $r$ does not divide $q-1=r^{e}-1$, the group $\tilde{R}$ has trivial intersection with $\Delta$, and induces a group $R \cong \tilde{R}$ on $H$. As the regular normal subgroup $M$ has order $q=r^{e}$, we have $M \leq R$, and $\tilde{R}$ contains an elementary abelian $r$-group $\tilde{M}$ of order $q$ acting regularly on $H$. In any case to be studied below, we will either show that $\tilde{M}$ acts trivially on the set of "horizontal" lines (parallel to $H$, corresponding to the circles touching $K$ at $\infty$ ) or show that $\tilde{M}$ acts trivially on $L_{\infty}$ (the line at infinity, whose points correspond to circles passing through $\infty$ and through $o$ ). As $\tilde{M}$ fixes no point of $\mathbb{A}_{\infty}$, both observations yield that $\tilde{M}$ induces a group of translations of $A_{\infty}$. These translations fix the line $H$, so they are translations along $H$, i.e., their center is the point at infinity corresponding to the parallel class of $H$. Together with the group $V$ of vertical translations contained in the elation group of $\mathscr{L}$, these translations secure that the projective closure $\mathbb{P}_{\infty}$ is a translation plane. As the dual of $\mathbb{P}_{\infty}$ is a translation plane anyway (see 1.1 , the assertion follows.

We consider first the case where $r=2$. As no involution in $\tilde{M}$ fixes any point in $\mathbb{A}_{\infty}$, we obtain that these involutions induce translations of $\mathbb{A}_{\infty}$, cf. 1.3 .

Now assume that $r$ is odd. If $q$ is not the square of a Mersenne prime then there exists a Zsigmondy prime $z$ for the field $\mathbb{F}_{q}$, i.e., a prime divisor $z$ of $q-1=r^{e}-1$ such that every irreducible linear representation of a cyclic group of order $z$ over $\mathbb{F}_{r}$ has dimension $e$, and induces a semiregular action on the set of nonzero vectors. See [40], cf. [35, Section 4]. As $\Gamma$ acts two-transitively on $H$, the order of $\Gamma$ is divisible by $z$, and there exists a cyclic subgroup $Z$ of order $z$ in $\Gamma$.

If $Z$ acts trivially on $H$ then $Z$ acts non-trivially on the additive group of the kernel $F$ of the translation plane dual to $\mathbb{P}_{\infty}$. As $z$ is a Zsigmondy prime, we obtain $F \cong \mathbb{F}_{q}$, and (the dual of) $\mathbb{P}_{\infty}$ is Desarguesian.

So assume that $Z$ acts non-trivially on $H$. Then $Z$ acts semi-regularly on $\tilde{M}$, and the set $[Z, \tilde{M}]$ of commutators coincides with $\tilde{M}$. We study the action of $Z$ on the line $L_{\infty}$ at infinity for $\mathbb{A}_{\infty}$. If $Z$ acts trivially on $L_{\infty}$, then $\tilde{M}=[Z, \tilde{M}]$ also acts trivially on $L_{\infty}$.

Now assume that $Z$ acts non-trivially on $L_{\infty}$. We consider the action of the group $Z \tilde{M}$ by conjugation on the quotient $E_{\infty} / V$; that action is equivalent to the action on $L_{\infty}-\{v\}$ where $v$ is the point at infinity belonging to the parallel class formed by the generators of $\mathscr{L}$ in $\mathbb{A}_{\infty}$. The groups $\tilde{M}$ and $E_{\infty} / V$ are $r$-groups. Therefore, the centralizer $U$ of $\tilde{M}$ in $E_{\infty} / V$ is not trivial. As a $Z$-module, the group $E_{\infty} / V$ splits as a direct sum of irreducible modules (by Maschke's Theorem), and at least one of these is not trivial. Such a submodule has order $q$ because $z$ is a Zsigmondy prime. Thus $E_{\infty} / V$ coincides with its non-trivial $Z$-submodule $U$. As $U$ corresponds to the fixed points of $\tilde{M}$ on $L_{\infty}-\{v\}$, we obtain again that $\tilde{M}$ acts trivially on $L_{\infty}$.

It remains to discuss the case where we do not have any Zsigmondy primes; as we have already dealt with the even order case, we then have that $q$ is the square of a Mersenne prime $r=2^{m}-1$. As $q=r^{2}$ divides the order of $\Gamma_{0}$, we apply 1.13 and obtain that $\mathscr{L}$ is Miquelian. 
3.3 Corollary. In $\mathbb{P}_{\infty}$, let $L_{\infty}$ denote the line at infinity for $\mathbb{A}_{\infty}$, let $u$ denote the point at infinity for $H$, and let $v$ denote the point at infinity for the vertical lines (i.e., the generators). Let $h$ be any point on $H$. Then the homology group $\operatorname{Aut}\left(\mathbb{P}_{\infty}\right)_{[x, Y]}$ is cyclic, for each anti-flag $(x, Y) \in$ $\left\{\left(h, L_{\infty}\right),(u,[h]),(v, H)\right\}$.

Proof. The plane $\mathbb{P}_{\infty}$ has Lenz type at least $\mathrm{V}$ by 3.2 . Therefore, that plane is coordinatized by a semifield (division ring), see [16, 6.9], and the homology groups in questions are isomorphic to multiplicative groups of the semi-nuclei of that semifield (see [16, 8.2]). These semi-nuclei are skew fields in general, and have cyclic multiplicative groups in the finite case.

\subsection{Lemma. The cover $\tilde{M}$ of the socle $M$ acts trivially on the generator [ $[$ ].}

Proof. We already know that $\tilde{M} \cong M$ is elementary abelian of order $q$. If there exists a Zsigmondy prime $z$ for $\mathbb{F}_{q}$ then pick a subgroup $Z$ of order $z$ in the stabilizer $\Gamma_{o}$.

If $Z$ acts trivially on [o] then $Z$ acts faithfully on $H$, and thus also faithfully on $M$. Then $\tilde{M}=$ $[Z, \tilde{M}]$ acts trivially on $[\infty]$, as well.

If $Z$ acts non-trivially on [o] we consider the centralizer $U$ of $\tilde{M}$ in $E / E_{\infty}$. As both $\tilde{M}$ and $E / E_{\infty}$ are $r$-groups, the group $U$ is not trivial. Since $E / E_{\infty}$ acts regularly on [ $\infty$ ], the action of $Z \tilde{M}$ by

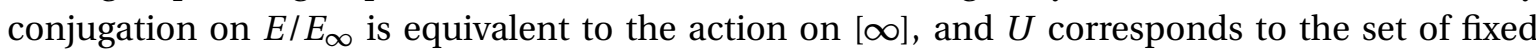

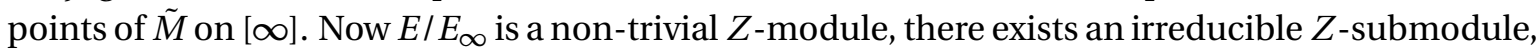
and that submodule coincides with $E / E_{\infty}$ because $Z$ is a Zsigmondy subgroup. The centralizer $U$ is also a $Z$-submodule, and we obtain $U=E / E_{\infty}$. Thus $\tilde{M}$ acts trivially on [ $[$ ], as claimed.

If there is no Zsigmondy prime we either have $q=2^{6}$ (and this case is dealt with in 6.3 below), or $q=r^{2}$ with a Mersenne prime $r=2^{m}-1$. In the latter case $\mathscr{L}$ is Miquelian 1.1 3, see 6.3 , and 1.2 applies.

3.5 Remark. An analogue of our result 3.2 has been proved by Ganley-Jha [11]: every finite translation plane with a group of automorphisms fixing one point $x$ on the line $L_{\infty}$ at infinity and acting two-transitively on $L_{\infty}-\{x\}$ is a semifield plane. See also [14].

\section{The odd order case}

4.1 Theorem. Let $\mathscr{L}$ be an elation Laguerre plane of odd order. If there exists a point $\infty$ such that $\operatorname{Aut}(\mathscr{L})_{\infty}$ acts two-transitively on $\mathscr{G} \backslash\{\infty\}$ then the affine plane $\mathbb{A}_{\infty}$ is Desarguesian, and $\mathscr{L}$ is Miquelian.

Proof. Without loss, we may assume that the (odd) order $q$ of $\mathscr{L}$ is neither a prime nor the square of a (Mersenne) prime, see 1.13 . So there exists a Zsigmondy prime, and 3.4 yields that $\tilde{M}$ acts trivially on [o]. By 3.2 the group $\tilde{M}$ acts faithfully as a group of translations on $A_{\infty}$. In [36] we have proved that this means that $\mathbb{P}_{\infty}$ admits at least $q-1$ different shift groups (induced by conjugates of $\tilde{M}$ under $\Delta$ ). So $\mathbb{P}_{\infty}$ is Desarguesian ([22, 9.4], cf. [36]), and $\mathscr{L}$ is Miquelian (see $1.1[3$ ).

\section{Complements}

We study the stabilizers $\Gamma_{o}$ and $G_{o}$, respectively, for some point $o \in H$. Note that we have a semidirect product $G=M \rtimes G_{o}$ where the action of $G_{o}$ by conjugation on $M$ is linear over the prime 
field $\mathbb{F}_{r}$. The possibilities for $G_{o}$ are quite restricted, see Table 2 (compiled ${ }^{1}$ from [17] and [13], cp. [5] or [19, XII, 7.5]. Note that (after 4.1) only the cases with even $q$ are relevant for the present section.

\begin{tabular}{|c|c|c|}
\hline & eligible $q=r^{e}$ & reference, remarks \\
\hline$G_{o} \leq \Gamma \mathrm{L}(1, q)$ & & \\
\hline $\mathrm{SL}\left(d, r^{e / d}\right) \unlhd G_{o} \leq \Gamma \mathrm{L}\left(d, r^{e / d}\right)$ & $q=r^{d c}$ with $d>1$ & 3.1 \\
\hline $\operatorname{Sp}\left(2 b, r^{e / 2 b}\right) \unlhd G_{o}$ & $q=r^{2 b c}$ with $b>1$ & 3.1 \\
\hline $\mathrm{G}_{2}\left(2^{a}\right) \unlhd G_{o}$ & $q=2^{6 a}, a>1$ & 5.1 \\
\hline $\mathrm{G}_{2}(2)^{\prime} \unlhd G_{o}$ & $q=2^{6}$ & 5.1, $\mathrm{G}_{2}(2)^{\prime} \cong \operatorname{PSU}\left(3,3^{2}\right)$ \\
\hline$G_{o} \cong \mathrm{A}_{s}$ & $q=2^{4}, s \in\{6,7\}$ & 5.1 \\
\hline $\mathrm{SL}(2,3) \unlhd G_{o}$ & $\begin{array}{l}q=f^{2} \text { with } f \in\{, 7,11,23\} \\
q=f^{2} \text { with } f \in\{9 .\end{array}$ & $\mathrm{Q}_{8} \unlhd \mathrm{SL}(2,3)$ \\
\hline$G_{o} \cong \operatorname{SL}(2,13)$ & $\begin{array}{l}q=J^{-} \text {with } J \in\{9,11,19,29,59\} \\
q=3^{6}\end{array}$ & \\
\hline$N \unlhd G_{O}$ & $q=3^{4}, N$ extraspecial of order $2^{5}$ & $\begin{array}{l}G_{o} / N \cong A \leq \mathrm{S}_{5} \\
N \cong \mathrm{Q}_{8} \cdot \mathrm{D}_{4}\end{array}$ \\
\hline
\end{tabular}

Table 2: Complements of abelian socles in two-transitive groups of degree $q=r^{e}$

\subsection{Theorem. The stabilizer $G_{o}$ is contained in $\Gamma \mathrm{L}\left(1, \mathbb{F}_{q}\right)$.}

Proof. After 4.1 and 1.2, it only remains to study the case where $r=2$. In 3.1, we have shown that $d=2=f$ if $G_{o}$ has a normal subgroup isomorphic to $\operatorname{SL}(d, f)$ or to $\operatorname{Sp}(d, f)$, where $q=f^{d}$ for some $d>1$. The plane is then the Miquelian plane of order 4 , and $G \cong \mathrm{S}_{4} \cong \operatorname{A\Gamma L}(1,4) \cong \operatorname{AGL}(2,2)$.

Inspection of Table 2 leaves us with two cases that we exclude in the sequel; namely a normal subgroup isomorphic to $\mathrm{G}_{2}(q)^{\prime}$ in $G_{o}$, or $G_{o} \cong \mathrm{A}_{m}$ with $m \in\{6,7\}$ and $q=16$.

The group $\mathrm{G}_{2}(f)$ is the automorphism group of the (split) octonion algebra $\mathbb{O}_{f}$ over $\mathbb{F}_{f}$. See [28] for an introduction of octonion algebras in the context of composition algebras, over arbitrary fields. The group $\mathrm{G}_{2}(f)$ is also discussed in [38, 4.3]. The octonion algebra can be constructed by repeated doubling (cf. [28, 1.5.3]), starting from any two-dimensional composition algebra over $\mathbb{F}_{f}$. We start from the quadratic extension field $\mathbb{F}_{f^{2}}$ and find $u \in \mathbb{O}_{f}$ such that $u$ has trace 1 and generates a subalgebra $\mathbb{F}_{f}(u)$ isomorphic to $\mathbb{F}_{f^{2}}$. The stabilizer of $u$ in $\mathrm{G}_{2}(f)$ is then isomorphic to $\mathrm{SU}\left(3, f^{2}\right)$, cf. [38, 4.3.6], and acts on the orthogonal complement $U$ of $\mathbb{F}_{f}(u)$ in $\mathbb{O}_{f}$. This action is linear over $\mathbb{F}_{f}(u) \cong \mathbb{F}_{f^{2}}$, and equivalent to the standard action of $\operatorname{SU}\left(3, f^{2}\right)$ on $\left(\mathbb{F}_{f^{2}}\right)^{3}$. Therefore, each involution in such a stabilizer fixes either $f^{2}$ (if $f$ is odd) or $f^{4}$ (if $f$ is even) of the $f^{6}$ vectors in $U$. Note that these involutions are in fact contained in the commutator group; this is clear if $f>2$ because then $\mathrm{G}_{2}(f)$ is simple (and coincides with its commutator subgroup), and also true if $f=2$ because the Sylow 2-subgroup of $\mathrm{SU}(3,4)$ is a quaternion group (cf. [18, 10.12 b), 10.14]).

If $f=2^{a}$ then the polar form corresponding to the norm on $\mathbb{O}_{f}$ is alternating, and 1 is contained in $1^{\perp}$ but not in $U$. Thus $\mathrm{G}_{2}(f)$ acts on the quotient of $1^{\perp}$ modulo the center of $\mathbb{O}_{f}$. This is the linear representation of $\mathrm{G}_{2}(f)$ on $\mathbb{F}_{2^{6}}^{6}$ that gives rise to the semidirect product $\mathbb{F}_{2}^{6 a} \rtimes \mathrm{G}_{2}\left(2^{a}\right)$ acting two-transitively on $2^{6 a}$ points. The action of the stabilizer of $u$ on that quotient is equivalent to the action on $U$ because the center of $\mathbb{O}_{f}$ intersects $U$ trivially.

\footnotetext{
${ }^{1}$ Note that the list in [6 Table 7.3] omits the semidirect product $\mathbb{F}_{9}^{2} \rtimes \operatorname{SL}(2,5)$ ). The list in [10 Table 7.1] gives wrong generators (surely, the matrix $\left(\begin{array}{cc}0 & -1 \\ -1 & 0\end{array}\right)$ has eigenvalue 1 , and should not occur in a sharply transitive linear group). Deleting one of the minus signs in generator $a$ as given in [10, Table 7.1] yields the generators as given by Zassenhaus 39 Satz 17].
} 
If $\mathrm{G}_{2}\left(2^{a}\right)$ would occur as a normal subgroup of $G_{o}$ then the involutions in $\mathrm{G}_{2}\left(2^{a}\right)$ would be induced by involutions in $\Gamma_{o}$ because the kernel $\Delta_{K}$ has odd order; and these involutions would fix $2^{4 a}$ of the $2^{6 a}$ points on the affine line $H$. This gives a contradiction to 1.3 .

Now assume that $G_{o}$ is isomorphic to $\mathrm{A}_{6}$ or $\mathrm{A}_{7}$, and $q=16$. (This case has been excluded by [33 which involves a computer search - we give a direct argument here.) Let $\tilde{T}$ be a Sylow 2-subgroup of $\Gamma_{o}$. Since the order of $\Delta_{K}$ is odd, we see that $\tilde{T}$ acts faithfully on $H$, inducing a Sylow 2-subgroup $T$ of $G_{o}$. In particular, the group $T$ is isomorphic to a dihedral group of order 8. Furthermore, $T$ fixes at least 3 generators of $\mathscr{L}$, and on each such generator at least 2 points. Hence, the collection of fixed points, fixed circles and fixed generators forms a Laguerre plane $\mathscr{L}_{T}$ by 1.6

Let $\sigma$ be the central involution in $T$. Since $\sigma$ induces a Baer involution in the derived plane at a fixed point, we see that the geometry $\mathscr{L}_{\sigma}$ of fixed elements of $\sigma$ is a Laguerre plane of order 4. Furthermore, $\mathscr{L}_{\sigma}$ contains $\mathscr{L}_{T}$ as a subplane. The group $T$ centralizes $\sigma$ and thus induces a group of automorphisms of $\mathscr{L}_{\sigma}$.

Each Laguerre plane of order 4 is Miquelian. In the full automorphism group of the Miquelian Laguerre plane of order 4 we find that the pointwise stabilizer of a subplane of order 2 is a group of order 2. Therefore, there must be a subgroup $H$ of order 4 in $T$ that fixes each point of $\mathscr{L}_{\sigma}$. Let $x$ be a point moved by $\sigma$. Since $\mathscr{L}_{\sigma}$ contains $4^{3}>16$ circles, there must be at least two distinct circles of $\mathscr{L}_{\sigma}$ that intersect $[x]$ in the same point $w$. The intersection of these two circles is a set $\left\{w, w^{\prime}\right\}$ of size at most 2 , and invariant under $H$. But $H$ has order 4 so that the stabilizer $H_{w}$ is nontrivial. We consider the projective closure $\mathbb{P}_{\infty}$ of the derived affine plane at $\infty$. The group $H_{w}$ fixes a Baer subplane $\mathbb{B}$ of $\mathbb{P}_{\infty}$ pointwise, and also the point $w$ which lies outside $\mathbb{B}$. This is impossible because $\mathbb{B}$ is a maximal subplane.

\section{Describing circles in the case of even order}

From 3.2 we know that $\mathbb{A}_{\infty}$ is a translation plane of Lenz type at least V. So we coordinatize it using a semifield $(X,+, \cdot)$ such that $\mathbb{A}_{\infty}$ has point set $X^{2}$, the point $o$ becomes $\left(\begin{array}{l}0 \\ 0\end{array}\right)$, and the lines are either

- verticals of the form $[c]=\left\{\left(\begin{array}{l}c \\ y\end{array}\right) \mid y \in X\right\}$ (these are generators of the Laguerre plane $\mathscr{L}$ ), or

- of the form $[m, b]:=\left\{\left(\begin{array}{c}x \\ m \cdot x+b\end{array}\right) \mid x \in X\right\}$ with $m, b \in X$.

Then

$$
\xi_{v, z, w}: X^{2} \rightarrow X^{2}:\left(\begin{array}{l}
x \\
y
\end{array}\right) \mapsto\left(\begin{array}{c}
x+v \\
y+z+w \cdot x
\end{array}\right)
$$

is an automorphism of $\mathbb{A}_{\infty}$, for any $v, z, w \in X$. The set $\Xi:=\left\{\xi_{v, z, w} \mid v, z, w \in X\right\}$ forms a nilpotent group of order $q^{3}$, multiplication is given by $\xi_{a, c, b} \xi_{v, z, w}=\xi_{v+a, z+c+b \cdot v, w+b}$.

From 3.2 we know that $\Gamma$ contains the group $\tilde{M}$ of translations along $H$. In the present description, we note $\tilde{M}=\left\{\xi_{v, 0,0} \mid v \in X\right\}$ and $E_{\infty}=\left\{\xi_{0, z, w} \mid z, w \in X\right\}$, so $\Xi=E_{\infty} \tilde{M}$ is contained in $\operatorname{Aut}(\mathscr{L})$.

6.1 Lemma. Assume that $q$ is even. Consider a point $d \in[\infty]-\{\infty\}$ and the circle $C_{d}$ through $d$ touching $K$ in $o$. Then the set $V_{d}:=\left\{v \in X \mid \xi_{v, 0,0}\right.$ fixes $\left.d\right\}$ forms a subgroup of $(X,+)$, and there exists a function $f_{d}: X \rightarrow X$ such that the following hold.

1. $C_{d}=\{d\} \cup\left\{\left(\begin{array}{c}x \\ f_{d}(x)\end{array}\right) \mid x \in X\right\}$.

2. For each $(x, v) \in X \times V_{d}$ we have $f_{d}(x+v)=f_{d}(x)+f_{d}(v)$.

3. The restriction $\left.f_{d}\right|_{V_{d}}:\left(V_{d},+\right) \rightarrow(X,+)$ is an injective homomorphism. 
Proof. The set $V_{d}$ is closed under addition because it parameterizes the stabilizer $\tilde{M}_{d}$, and $\xi_{v, 0,0} \xi_{y, 0,0}=$ $\xi_{v+y, 0,0}$. Since every generator meets $C:=C_{d}$ precisely once, there is a function $f=f_{d}: X \rightarrow X$ such that

$$
C=\{d\} \cup\left\{\left(\begin{array}{c}
x \\
f(x)
\end{array}\right) \mid x \in X\right\}
$$

and $f(0)=0$ because $C$ passes through $o=\left(\begin{array}{l}0 \\ 0\end{array}\right)$.

Consider $v \in V_{d}$. The group $E_{\infty}$ fixes each point in [ $\infty$ ], and acts semi-regularly and then sharply transitively on $\mathscr{C}_{d}$. Therefore, we find $z=z_{\nu}$ and $w=w_{\nu} \in X$ (depending on $v$, possibly) such that $\xi_{v, 0,0}(C)=\xi_{0, z, w}(C)$. This means

$$
\{d\} \cup\left\{\left(\begin{array}{l}
x+\nu \\
f(x)
\end{array}\right) \mid x \in X\right\}=\xi_{\nu, 0,0}(C)=\xi_{0, z, w}(C)=\{d\} \cup\left\{\left(\underset{s(s)+z_{v}+w_{v} \cdot s}{ }\right) \mid s \in X\right\} .
$$

We obtain $f(x+v)+z_{v}+w_{v} \cdot(x+v)=f(x)$ for all $x \in X$. Specializing $x=0$ we find $f(v)=-z_{v}-w_{v} \cdot v$. Thus $f(x+v)+w_{v} \cdot x=f(x)+f(v)$. Specializing $x=-v$ we find $-w_{\nu} \cdot v=f(-v)+f(\nu)$.

Up to this point, we did not use our assumption that $q$ is even. Now we apply this assumption to the last equation and obtain $w_{v}=0$ for each $v \neq 0$. Thus $f(x+v)=f(x)+f(v)$ holds whenever $v \in V_{d}$. In particular, the restriction $\left.f\right|_{V_{d}}$ is additive. As $C$ touches $K$ in $o$, the kernel of this additive map is trivial.

6.2 Corollary. If $d$ is fixed by $\tilde{M}$ then the map $f_{d}$ in 6.1 is an automorphism of $(X,+)$.

6.3 Theorem. The cover $\tilde{M}$ of the socle acts trivially on the generator $[\infty]$ if $q$ is even.

Proof. Aiming at a contradiction, we assume that there exists $d \in[\infty]$ such that $\tilde{M}_{d} \neq \tilde{M}$. Then $d \neq \infty$, and $\tilde{M}_{d}$ is not trivial because $|\tilde{M}|=q>q-1=|[\infty]-\{\infty\}|$.

Pick $y \in X-V_{d}$, and let $D$ be the circle through $e:=\xi_{y, 0,0}(d)$ that touches $K$ in $o$. From 6.1 we have the function $f_{e}: X \rightarrow X$ such that $D=\{e\} \cup\left\{\left(\begin{array}{c}x \\ f_{e}(x)\end{array}\right) \mid x \in X\right\}$. Now $\xi_{y, 0,0}=\xi_{y, 0,0}^{-1}$ moves $D$ to a circle through $d$, and there exist $u$ and $s$ in $X$ (depending on $y$ ) such that $\xi_{0, u, s}\left(\xi_{y, 0,0}(D)\right)=C$. We obtain $f_{e}(x)+u+s \cdot(x+y)=f_{d}(x+y)$, for all $x \in X$. Specializing $x=0$ we find $u+s \cdot y=f_{d}(y)$. Returning to the general case, we obtain $f_{e}(x)+f_{d}(y)+s \cdot x=f_{d}(x+y)$, for all $x \in X$. From 6.1 we infer $f_{e}(v)+s \cdot v=f_{d}(v)$ for all $v \in V_{d}$.

Now $\xi_{0,0, s} \in E_{\infty}$ fixes $d$, and $\xi_{0,0, s}(C)=\{d\} \cup\left\{\left(\begin{array}{c}x \\ f_{d}(x)+s \cdot x\end{array}\right) \mid x \in X\right\}$. The intersection of that circle with $D$ contains the set $\left\{\left(\begin{array}{c}v \\ f_{e}(v)\end{array}\right) \mid v \in V_{d}\right\}$ but the two circles are different because they [o] in different points (namely $d$ and $\xi_{y, 0,0}(d)$, respectively). This yields $\left|V_{d}\right| \leq 2$, and $\left|V_{d}\right|=2$ because $\tilde{M}_{d}$ is not trivial.

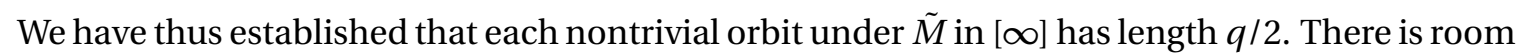

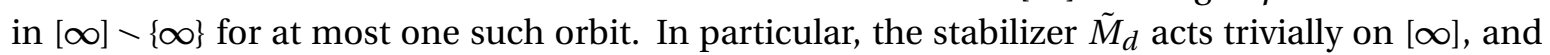
forms a normal subgroup in $\Gamma$ because $\tilde{M}_{d}$ is the intersection of $\tilde{M}$ with the kernel of the action of $\Gamma$ on the fixed generator $[\infty]$. This contradicts the fact that $G_{o}$ acts irreducibly on $M$.

6.4 Corollary. For each $d \in[\infty] \backslash\{\infty\}$, the map $f_{d}$ in 6.1 is an automorphism of $(X,+)$.

The information collected so far is far from sufficient to determine the Laguerre plane $\mathscr{L}$. There are many candidates for $f_{d} \in \operatorname{Aut}(X,+)$, and it is still conceivable that $f_{d}$ depends in a substantial way on $d$. 


\section{The Desarguesian case}

By [8] a semifield plane that admits an autotopism group that is transitive on at least one side of the autotopism triangle is a generalized twisted field plane unless the order is 64 . It readily follows from the description of generalized twisted fields (for example, see [3, \$2]) that a generalized twisted field of order $2^{e}$ cannot be proper when $e$ is a prime number or the square of a prime number. Indeed, the pre-semifield that leads to a generalized twisted field of order $2^{e}$ is described by two automorphisms of $\mathbb{F}_{2^{e}}$, given as $x \mapsto x^{2^{a}}$ and $x \mapsto x^{2^{b}}$ where $1 \leq a, b<e$ and $a \neq b$, and a suitable field element $c$; the latter exists if and only if the greatest common divisor of $e, a$ and $b$ is greater than 1. Hence we obtain:

6.5 Theorem. If $q=2^{e}$ with e either a prime or the square of a prime then $\mathbb{A}_{\infty}$ is Desarguesian.

A Desarguesian derivation restricts the possibilities for the Laguerre plane.

6.6 Theorem. If $q$ is even and $\mathbb{A}_{\infty}$ is Desarguesian, then $\mathscr{L}$ is ovoidal over a translation oval.

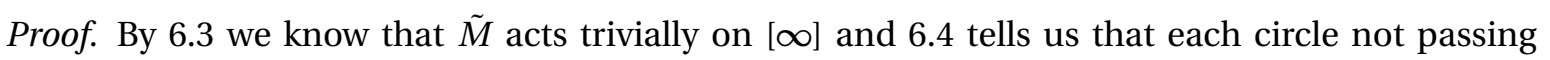
through $\infty$ comes from a translation oval in $\mathbb{P}_{\infty}$. These ovals are known explicitly (see [25] and [15]); we obtain: For each circle $C$ touching $K$ in $o$ there is a field element $a \in \mathbb{F}_{q}$ and a generator $\theta_{a}$ of Aut $\left(\mathbb{F}_{q}\right)$ such that $C-[\infty]=\left\{\left(\begin{array}{c}x \\ a x^{\theta_{a}}\end{array}\right) \mid x \in \mathbb{F}_{q}\right\}$. As $\theta_{a}$ is a power of the Frobenius automorphism, there exists a positive integer $m_{a}<e$ such that $x^{\theta_{a}}=x^{2^{m a}}$, and $m_{a}$ is relatively prime to $e$ because $\theta_{a}$ generates Aut $\left(\mathbb{F}_{q}\right)$. Mapping $x$ to $x^{\theta_{a}} / x$ gives an endomorphism $\theta_{a}-1$ of $\mathbb{F}_{q}^{\times}$. As $\theta_{a}$ generates Aut $\left(\mathbb{F}_{q}\right)$, its field of fixed elements is the prime field $\mathbb{F}_{2}$. Therefore, the endomorphism $\theta_{a}-1$ has trivial kernel, and is a bijection of $\mathbb{F}_{q}^{\times}$.

Extending the usual representation of dual translation planes, a description of elation Laguerre planes in terms of a matrix-valued map was developed in [29, Theorem 3]. In particular, we know that the elation group $E$ consists of all maps $\left(\begin{array}{l}x \\ y\end{array}\right) \mapsto\left(\begin{array}{c}x \\ y+f(x)\end{array}\right)$ where $f$ describes a circle of $\mathscr{L}$. It thus follows that $x \mapsto \alpha x^{\theta_{\alpha}}+\beta x^{\theta_{\beta}}$ describes a circle, for all $\alpha, \beta \in \mathbb{F}_{q}$. As that circle touches $K$ in $o$, there exists $\gamma \in \mathbb{F}_{q}$ such that $\alpha x^{\theta_{\alpha}}+\beta x^{\theta_{\beta}}=\gamma x^{\theta_{\gamma}}$ for all $x \in \mathbb{F}_{q}$. As the exponents are less than $q$, that polynomial identity has to be trivial. We find $\theta_{\alpha}=\theta_{\beta}=\theta_{\gamma}$ and $\alpha+\beta=\gamma$; in particular, the automorphism $\theta:=\theta_{\alpha}$ does not depend on $\alpha$. Consequently, the traces of circles in $\mathscr{L}$ on $\mathbb{A}_{\infty}$ are of the form

$$
C_{a, b, c}^{\prime}=\left\{\left(\begin{array}{c}
x \\
a x^{\theta}+b x+c
\end{array}\right) \mid x \in \mathbb{F}_{q}\right\}
$$

where $a, b, c \in \mathbb{F}_{q}$. This incidence structure on $\mathbb{F}_{q} \times \mathbb{F}_{q}$ extends to an ovoidal Laguerre plane by amending each circle with a point on $[\infty]$. In fact, there are two choices (see [32, p. $93 \mathrm{f}]$ ): either each trace $C_{a, b, c}^{\prime}$ as above is extended by $\left(\begin{array}{c}\infty \\ a\end{array}\right)$, or each trace $C_{a, b, c}^{\prime}$ is extended by $\left(\begin{array}{c}\infty \\ b\end{array}\right)$.

In the present situation, however, the introduction of coordinates in $A_{\infty}$ already identifies the circles that pass through $\infty$; their traces are of the form $C_{0, b, c}^{\prime}$ (i.e., we have $a=0$ ). We now consider the bundle $\mathscr{B}$ of circles that touch the circle $K$ at $o$. One of them is $K$; every other circle in this bundle has a trace $C_{a, b, c}^{\prime}$ where $a \neq 0$. We know that $c=0$ because $o \in C_{a, b, c}^{\prime}$. But then $b=0$ because otherwise the image of $b / a$ under $(\theta-1)^{-1}$ would give a second point (apart from $o$ ) in $C_{a, b, 0}^{\prime} \cap K$. This shows that the traces of the circles in $\mathscr{B}$ are just those in $\left\{C_{a, 0,0}^{\prime} \mid a \in \mathbb{F}_{q}\right\}$. Thus there is a bijection $\varphi$ from $\mathbb{F}_{q}$ onto [o] such that $C_{a, b, c}^{\prime} \cup\left\{\left(\begin{array}{c}\infty \\ \varphi(a)\end{array}\right)\right\}$ is a circle, for each triple $(a, b, c) \in \mathbb{F}_{q}^{3}$. It is obvious from the action of $E$ that $\varphi$ is additive. Without loss of generality, we may thus assume $\varphi=$ id, and we obtain that $\mathscr{L}$ is isomorphic to the ovoidal Laguerre plane over the translation oval in $\mathbb{P}_{\infty}$ whose trace in $\mathbb{A}_{\infty}$ is $\left\{\left(\begin{array}{c}x \\ x^{\theta}\end{array}\right) \mid x \in \mathbb{F}_{q}\right\}$; that oval's unique point at infinity corresponds to the parallel class of vertical lines (induced by generators of $\mathscr{L}$ ). 
6.7 Corollary. If the order of $\mathscr{L}$ is $q=2^{e}$ where $e$ is either a prime number or the square of a prime number, then $\mathscr{L}$ is ovoidal over a translation oval.

6.8 Remarks. The smallest integer $e>1$ which is neither a prime nor the square of a prime is $e=6$, so the smallest unresolved case is $q=64$. This is the exceptional case in [8]. The authors of [27] conducted a computer search for semifields of order 64 . They found a total of 332 such semifields up to isotopism, falling into 80 orbits under an action of the symmetric group $\mathrm{S}_{3}$. However, only four of those 80 classes contain semifields admitting a transitive action of the autotopism group on at least one side of the autotopism triangle. These are represented by the field, the twisted field, Knuth's semifield (of type 5), and a previously unknown semifield (number XIV in their list), respectively.

There remains the question whether sufficiently many translation ovals can be found in a plane over one of the latter three types. Some translation ovals in semifield planes were constructed by Jha and Wene, see [20].

Acknowledgement. The present investigation has been conducted during a stay of the second author as a Visiting Erskine Fellow at the University of Canterbury, Christchurch, New Zealand.

\section{References}

[1] M. Aschbacher, Finite group theory, Cambridge Studies in Advanced Mathematics 10, Cambridge University Press, Cambridge, 2nd edn., 2000, ISBN 0-521-78145-0; 0-521-78675-4. MR 1777008 (2001c:20001). Zbl 0997.20001.

[2] J. Bamberg and T. Penttila, A classification of transitive ovoids, spreads, and $m$ systems of polar spaces, Forum Math. 21 (2009), no. 2, 181-216, ISSN 0933-7741. doi:10.1515/FORUM.2009.010. MR 2503303. Zbl 1191.05029,

[3] M. Biliotti, V. Jha, and N. L. Johnson, The collineation groups of generalized twisted field planes, Geom. Dedicata 76 (1999), no. 1, 97-126, ISSN 0046-5755, doi : 10.1023/A :1005089016092. MR 1699202 (2001d:51003).

[4] W. Burnside, Theory of groups of finite order, Cambridge University Press, Cambridge, 2nd edn., 1911. MR 0069818 (16,1086c). JfM 42.0151.02.

[5] P. J. Cameron, Finite permutation groups and finite simple groups, Bull. London Math. Soc. 13 (1981), no. 1, 1-22, ISSN 0024-6093, doi:10.1112/blms/13.1.1. MR 599634 (83m:20008). Zbl 0463.20003,

[6] P. J. Cameron, Permutation groups, London Mathematical Society Student Texts 45, Cambridge University Press, Cambridge, 1999, ISBN 0-521-65302-9; 0-521-65378-9. MR 1721031 (2001c:20008). Zbl 0922.20003

[7] Y. Chen and G. Kaerlein, Eine Bemerkung über endliche Laguerre- und Minkowski-Ebenen, Geometriae Dedicata 2 (1973), 193-194, ISSN 0046-5755, doi:10.1007/BF00147856. MR 0407718 (53 \#11489). Zbl 0274.50021.

[8] M. Cordero and R. F. Figueroa, Transitive autotopism groups and the generalized twisted field planes, in Mostly finite geometries (Iowa City, IA, 1996), Lecture Notes in Pure and Appl. Math. 190, pp. 191-196, Dekker, New York, 1997. MR 1463983. Zbl 0901.51009. 
[9] T. Czerwinski, Finite translation planes with collineation groups doubly transitive on the points at infinity, J. Algebra 22 (1972), 428-441, ISSN 0021-8693, doi: 10.1016/0021-8693(72)90159-7. MR 0313933 (47 \#2485). Zbl 0243.50011.

[10] J. D. Dixon and B. Mortimer, Permutation groups, Graduate Texts in Mathematics 163, Springer-Verlag, New York, 1996, ISBN 0-387-94599-7, MR 1409812 (98m:20003). Zbl 0951.20001 ,

[11] M. J. Ganley and V. Jha, On translation planes with a 2-transitive orbit on the line at infinity, Arch. Math. (Basel) 47 (1986), no. 4, 379-384, ISSN 0003-889X, doi :10.1007/BF01191367. MR 866529 (88a:51012). Zbl 0596.51002.

[12] D. G. Glynn and G. F. Steinke, Laguerre planes of even order and translation ovals, Geom. Dedicata 51 (1994), no. 2, 105-112, ISSN 0046-5755, doi : 10. 1007/BF01265322. MR 1289081 (95h:51022).

[13] C. Hering, Transitive linear groups and linear groups which contain irreducible subgroups of prime order, Geom. Dedicata 2 (1974), 425-460, ISSN 0046-5755 doi :10.1007/BF00147570. MR 0335659 (49 \#439). Z Zbl 0292.20045.

[14] Y. Hiramine, On finite affine planes with a 2-transitive orbit on $l_{\infty}$, J. Algebra 162 (1993), no. 2, 392-409, ISSN 0021-8693 doi:10.1006/jabr. 1993.1262. MR 1254783 (94m:20006). Zbl 0799.51005.

[15] J. W. P. Hirschfeld, Ovals in desarguesian planes of even order, Ann. Mat. Pura Appl. (4) 102 (1975), 79-89, ISSN 0003-4622, doi : 10.1007/BF02410598. MR 0358553. Zbl 0293.50015.

[16] D. R. Hughes and F. C. Piper, Projective planes, Graduate Texts in Mathematics 6, SpringerVerlag, New York, 1973, ISBN 978-0387900445. MR 0333959 (48 \#12278). Zbl 0484.51011.

[17] B. Huppert, Zweifach transitive, auflösbare Permutationsgruppen, Math. Z. 68 (1957), 126150, ISSN 0025-5874, do i : 10 . 1007/BF01160336. MR 0094386 (20 \#904). Zbl 0079.25502.

[18] B. Huppert, Endliche Gruppen. I, Grundlehren der Mathematischen Wissenschaften 134, Springer-Verlag, Berlin, 1967, ISBN 978-3540038252. MR 0224703 (37 \#302). Zbl 0217.07201.

[19] B. Huppert and N. Blackburn, Finite groups. III, Grundlehren der Mathematischen Wissenschaften 243, Springer-Verlag, Berlin, 1982, ISBN 3-540-10633-2. MR 662826 (84i:20001b). ZZbl 0514.20002.

[20] V. Jha and G. P. Wene, The structure of the central units of a commutative semifield plane, in Finite geometry and combinatorics (Deinze, 1992), London Math. Soc. Lecture Note Ser. 191, pp. 207-216, Cambridge Univ. Press, Cambridge, 1993, doi:10.1017/CB09780511526336.022. MR 1256278 (94i:51009). Zbl 0798.51004.

[21] W. M. Kantor, Homogeneous designs and geometric lattices, J. Combin. Theory Ser. A 38 (1985), no. 1, 66-74, ISSN 0097-3165, doi:10.1016/0097-3165(85)90022-6. MR773556 (87c:51007). Zbl 0559.05015.

[22] N. Knarr and M. J. Stroppel, Polarities of shift planes, Adv. Geom. 9 (2009), no. 4, 577-603, ISSN 1615-715X, doi:10.1515/ADVGEOM. 2009.028. MR 2574140. Zbl 1181.51003. 
[23] R. Löwen and U. Pfüller, Two-dimensional Laguerre planes with large automorphism groups, Geom. Dedicata 23 (1987), no. 1, 87-96, ISSN 0046-5755, doi:10.1007/BF00147394. MR 886777 (88g:51029). Zbl 0615.51007.

[24] D. Passman, Permutation groups, W. A. Benjamin, Inc., New York-Amsterdam, 1968. MR 0237627 (38 \#5908). Zbl 0179.04405.

[25] S. E. Payne, A complete determination of translation ovoids in finite Desarguian planes, Atti Accad. Naz. Lincei Rend. Cl. Sci. Fis. Mat. Natur. (8) 51 (1971), 328-331 (1972). MR 0337658. Zbl 0238.50018.

[26] S. E. Payne and J. A. Thas, Generalized quadrangles with symmetry, Simon Stevin 49 (1975/76), no. 1/2, 3-32, ISSN 0037-5454. MR 0419266 (54 \#7290a). Zbl 0328.50017.

[27] I. F. Rúa, E. F. Combarro, and J. Ranilla, Classification of semifields of order 64, J. Algebra 322 (2009), no. 11, 4011-4029, ISSN 0021-8693, doi:10.1016/j.jalgebra.2009.02.020. MR 2556135. Zbl 1202.12003.

[28] T. A. Springer and F. D. Veldkamp, Octonions, Jordan algebras and exceptional groups, Springer Monographs in Mathematics, Springer-Verlag, Berlin, 2000, ISBN 3-540-66337-1. MR 1763974 (2001f:17006). Zbl 1087.17001.

[29] G. F. Steinke, On the structure of finite elation Laguerre planes, J. Geom. 41 (1991), no. 1-2, 162179, ISSN 0047-2468, doi : 10.1007/BF01258517. MR 1116911 (92i:51026). Zbl 0737.51003

[30] G. F. Steinke, A remark on Benz planes of order 9, Ars Combin. 34 (1992), 257-267, ISSN 03817032. MR 1206567 (94g:51019). Zbl 0770.51006

[31] G. F. Steinke, Elation Laguerre planes of order $p^{2}$ that admit an automorphism group of order $p^{2}$ in the elation complement, Australas. J. Combin. 8 (1993), 77-98, ISSN 1034-4942, http: // aj c.maths .uq.edu .au/pdf/8/ajc-v8-p77.pdf. MR 1240148 (94h:51026). Zbl 0806.51005.

[32] G. F. Steinke, Finite Möbius near-planes, Australas. J. Combin. 18 (1998), 77-96, ISSN 1034-4942, http://ajc.maths.uq.edu.au/pdf/18/ajc-v18-p77.pdf. MR 1658293 (99g:51010).

[33] G. F. Steinke, Elation Laguerre planes of order 16 are ovoidal, J. Combin. Des. 14 (2006), no. 4, 313-323, ISSN 1063-8539, doi:10.1002/jcd.20076. MR2229882 (2007c:51001). Zbl 1099.51003 .

[34] G. F. Steinke and M. J. Stroppel, Finite elation Laguerre planes admitting a two-transitive group on their set of generators, Innov. Incidence Geom. 13 (2013), 207-223, ISSN 1781-6475, http://iig.ugent.be/online/13/volume-13-article-11-online.pdf. MR3173020. Zbl 1307.51005

[35] G. F. Steinke and M. J. Stroppel, Simple groups acting two-transitively on the set of generators of a finite elation Laguerre plane, Beitr. Algebra Geom. 56 (2015), no. 1, 285-298, ISSN 0138-4821, doi:10.1007/s13366-013-0169-z. MR 3305450. Zbl 06407148

[36] G. F. Steinke and M. J. Stroppel, Laguerre planes and shift planes, Preprint 2017-008, Fachbereich Mathematik, Universität Stuttgart, Stuttgart, 2017, http://www. mathematik. uni-stuttgart.de/preprints/downloads/2012/2012-008.pdf. 
[37] J. A. Thas, The $m$-dimensional projective space $S_{m}\left(M_{n}(\mathrm{GF}(q))\right)$ over the total matrix algebra $M_{n}(\mathrm{GF}(q))$ of the $n \times n$-matrices with elements in the Galois field $\mathrm{GF}(q)$, Rend. Mat. (6) 4 (1971), 459-532, ISSN 0034-4427. MR 0315585 (47 \#4134). Zbl 0233.05010.

[38] R. A. Wilson, The finite simple groups, Graduate Texts in Mathematics 251, Springer-Verlag London Ltd., London, 2009, ISBN 978-1-84800-987-5, doi:10.1007/978-1-84800-988-2. MR 2562037 (2011e:20018). Zbl 1203.20012.

[39] H. Zassenhaus, Über endliche Fastkörper, Abh. Math. Sem. Univ. Hamburg 11 (1935), 187-220, doi:10.1007/BF02940723, MR 3069653. Zbl 0011.10302, JfM 61.0126.01.

[40] K. Zsigmondy, Zur Theorie der Potenzreste, Monatsh. Math. Phys. 3 (1892), no. 1, 265-284, ISSN 0026-9255, doi : 10.1007/BF01692444, MR 1546236. JfM 24.0176.02.

\author{
Günter F. Steinke \\ Department of Mathematics and Statistics \\ University of Canterbury \\ Private Bag 4800 \\ Christchurch 8140 \\ New Zealand \\ Markus J. Stroppel \\ LExMath \\ Fakultät für Mathematik und Physik \\ Universität Stuttgart \\ D-70550 Stuttgart \\ Germany
}





\section{Erschienene Preprints ab Nummer 2016-001}

Komplette Liste: http://www.mathematik.uni-stuttgart.de/preprints

2017-009 Steinke, G.; Stroppel, M.: On elation Laguerre planes with a two-transitive orbit on the set of generators

2017-008 Steinke, G.; Stroppel, M.: Laguerre planes and shift planes

2017-007 Blunck, A.; Knarr, N.; Stroppel, B.; Stroppel, M.: Transitive groups of similitudes generated by octonions

2017-006 Blunck, A.; Knarr, N.; Stroppel, B.; Stroppel, M.: Clifford parallelisms defined by octonions

2017-005 Knarr, N.; Stroppel, M.: Subforms of Norm Forms of Octonion Fields

2017-004 Apprich, C.; Dieterich, A.; Höllig, K.; Nava-Yazdani, E.: Cubic Spline Approximation of a Circle with Maximal Smoothness and Accuracy

2017-003 Fischer, S.; Steinwart, I.: Sobolev Norm Learning Rates for Regularized Least-Squares Algorithm

2017-002 Farooq, M.; Steinwart, I.: Learning Rates for Kernel-Based Expectile Regression

2017-001 Bauer, B.; Devroye, L; Kohler, M.; Krzyzak, A.; Walk, H.: Nonparametric Estimation of a Function From Noiseless Observations at Random Points

2016-006 Devroye, L.; Györfi, L.; Lugosi, G.; Walk, H.: On the measure of Voronoi cells

2016-005 Kohls, C.; Kreuzer, C.; Rösch, A.; Siebert, K.G.: Convergence of Adaptive Finite Elements for Optimal Control Problems with Control Constraints

2016-004 Blaschzyk, l.; Steinwart, l.: Improved Classification Rates under Refined Margin Conditions

2016-003 Feistauer, M.; Roskovec, F.; Sändig, AM.: Discontinuous Galerkin Method for an Elliptic Problem with Nonlinear Newton Boundary Conditions in a Polygon

2016-002 Steinwart, I.: A Short Note on the Comparison of Interpolation Widths, Entropy Numbers, and Kolmogorov Widths

2016-001 Köster, I.: Sylow Numbers in Spectral Tables 